(C) 2007 IEEE. Reprinted, with permission, from Steven Su, Nonparametric Hammerstein Model Based Model Predictive Control for Heart Rate Regulation . Engineering in Medicine and Biology Society, 2007. EMBS 2007. 29th Annual International Conference of the IEEE, 2007. This material is posted here with permission of the IEEE. Such permission of the IEEE does not in any way imply IEEE endorsement of any of the University of Technology, Sydney's products or services. Internal or personal use of this material is permitted. However, permission to reprint/republish this material for advertising or promotional purposes or for creating new collective works for resale or redistribution must be obtained from the IEEE by writing to pubs-permissions@ieee.org. By choosing to view this document, you agree to all provisions of the copyright laws protecting it 


\title{
Nonparametric Hammerstein Model Based Model Predictive Control for Heart Rate Regulation
}

\author{
Steven W. Su ${ }^{a *}$, Shoudong Huang ${ }^{a}, \mathrm{Lu} \mathrm{Wang}^{b}$, Branko G. Celler $^{b}$, \\ Andrey V. Savkin ${ }^{b}$, and Ying Guo ${ }^{c}$
}

\begin{abstract}
A novel nonparametric Hammerstein model identification approach integrated with Model Predictive Control is presented for the regulation of heart rate during treadmill exercise. As the complexity of human's cardiovascular system, the model structure (or pre-knowledge) of exercise response of cardiovascular system are often unknown. This paper provides a nonparametric modeling method for the identification of Hammerstein model which only needs well designed experimental data. Specifically, based on the pseudo-random binary sequence data, we can not only decouple the identification of from input nonlinearity but also obtain the step response of dynamic linear part by directly using correlation analysis. The powerful Support Vector Regression is adopted to obtain a nonparametric description of the inverse of static nonlinearity in order to obtain an approximate linear model of the Hammerstein system. A linear MPC controller is designed for the approximated linear model to achieve desired tracking performance under physical and safety related constrained. A Hammerstein model has been identified based on well designed experimental data for heart rate responses for exercise. Based on the model, a constrained MPC controller is designed. Simulation results show that the proposed algorithm can achieve desired heart rate tracking performance.
\end{abstract}

\section{Index Terms}

$a$ The Faculty of Engineering, University of Technology, Sydney, Australia.

$b$ Human Performance Group, Biomedical Systems Lab, School of Electrical Engineering \& Telecommunications University of New South Wales,UNSW Sydney N.S.W. 2052 Australia.

c Autonoumous Systems Lab, CSIRO ICT Center, Australia.

* The corresponding author is with Faculty of Engineering, University of Technology, Sydney, Australia. Email: Steven.Su@uts.edu.au. Tel: +61 (2) 95147603.

This research was supported by the Australian Research Council (Grant DP0452186). 


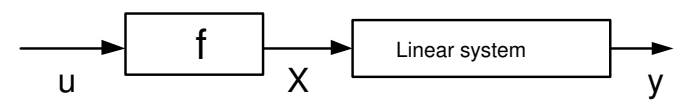

Fig. 1. A Hammerstein system.

Hammerstein model identification, Model Predictive control, Support Vector Regression, Heart rate control.

\section{INTRODUCTION}

There are well-developed theories for the control and identification of linear time invariant (LTI) systems. Most linear problems can be solved efficiently with standard numerical tools. However, as physical systems are nonlinear in nature, control and identification approaches for nonlinear system are practically important. A modest extension of linear model is Hammerstein model. See Figure 2.

The Hammerstein model can be described as a static nonlinear block followed by a dynamic linear system. Hammerstein models may account for nonlinear effects encountered in not only industrial processes [1] [2], but also physiological processes [3] [4]. For example, the electrically stimulated muscle [5], the upper limb [3], the muscle force [6], and the skeletal joints movements [7] can be effectively modeled by a nonlinear static element cascaded by a linear dynamic element.

Therefore, the modeling of Hammerstein model and a control method based on a Hammerstein model is of practical importance in engineering practice. This paper presented an integrated modeling and control approach based on Hammerstein models.

The modeling of Hammerstein model is an active research topic [] [] [] [] [] []. As far as the amount of the a priori information about the system is concerned, identification problems are either parametric or nonparametric [?]. In the nonparametric problem, the a priori information is much smaller then that of parametric problem. However, the nonparametric problem often better corresponds to real situations. For example, to model the cardiovascular responses, such as heart rate and respiration rate for exercise, the nonparametric identification approach should be more realistic because of the smaller information compared with the complexity of human bodies. Of course the choice of parametric or nonparametric model also depends on the requirements 
of a specific application. For model predictive control applications, this paper presents a new nonparametric identification approach based on support vector machine and stochastic method [] [] [] [] [] [].

As introduced in [8], the pseudo-random binary sequences (PRBS) are applied to decoupling the identification of linear dynamic part from nonlinearity. The correlation analysis is used to obtain the impulse response of the linear part directly. The powerful einsensitivity SVM approach is adopted to model the nonlinearity. It should be emphasized that the complexity of the nonlinear nonparametric SVM model can be well controlled by properly select a insensitive radius $\epsilon$.

One of the unique features of engineering control practice is the presence of operational constraints that limit the expected performance of the controlled system. These constraints arise from economic (quality) or safety considerations as well as from physical restrictions. Model Predictive Control offers a very attractive setting to provide disturbance rejection and setpoint tracking capabilities processes, in the presence of constraints.

Model predictive control (MPC) and PID control are the most popular approaches in industry. The main advantages of MPC is that it allows us to use the detailed knowledge of a process, in the form of a dynamic model, as an aid to controlling that process within the required constraints [?]. It is well known that almost all of the physical systems are subject to constraints on manipulated, state and output variables. In this study, we are trying to regulate hear rate of treadmill exercisers. Obviously, the speed and gradient of treadmill have up and low limitations. The capability of handling system constraints is a well-known advantage of MPC.

MPC has been well developed for linear systems. With the introduction of a dynamic nonlinear model, the MPC algorithm, the complexity of the predictive control problem increases significantly. The block oriented nonlinear model, Hammerstein model, is often employed to simplify the complexity. Most existing model predictive control methods for Hammerstein systems are based on direct inversion of the static nonlinearity combined with existing linear control approaches [9] [2] [?] . Similar strategy is adopted in this paper. Firstly, the approximation of the inversion of static nonlinearity is directly obtained by using $\epsilon$-insensitivity SVM. Then, the MPC controller is designed for the simplified linear model to achieve desired tracking performance under constraints. It should be noted that the inversion of the static nonlinearity cannot be achieved directly by using the method proposed in [10]. Another advantage of the proposed 
scheme is that it can directly using nonparametric model (step response), which can be obtained by our proposed correlation analysis based nonparametric model identification method.

In sport training, medical diagnosis, rehabilitation and analysis of cardio respiratory kinetics, automated exercise testing systems have revealed their growing importance [11] [12] [13]. These systems can fully implement programmed exercise and training protocols to achieve desired exercising and testing results. Another topic of this paper is the design of an automated heart rate regulation system for exercise on a motorized treadmill. Some commercial treadmills are already available which offer heart rate control. However, these normally use very simple control strategies, their control performance is poor. In this study, the proposed identification and control approach was applied for the automated heart rate regulation system design, and excellent tracking results are achieved. This is also the first report of SVM application in the cardiovascular system identification and control practice.

The paper is organized as follows. The details of SVM based identification and model predictive control approach are given in Section 2. Section 3 describes the identification of Hammerstein model of heart rate response for treadmill exercises based on the proposed approach. Section 4 presents simulation studies in the application of the proposed approach for the regulation of heart rate of treadmill exerciser. 
UTOMATED exercise testing systems have become increasingly important in sport train$A$ ing, medical diagnosis, rehabilitation and analysis of cardio respiratory kinetics [11] [12] [13]. These systems can fully implement programmed exercise and training protocols to achieve desired exercising and testing results. The major aim of this study is to develop a computer controlled treadmill system, which can control the heart rate of the subject according to a preset heart rate profile. Some commercial treadmills are already available which offer heart rate control. However, these normally use very simple control strategies, their control performance is poor and they have no mechanism for setting a desired heart rate profile. In this study we design a treadmill exercise system which can automatically control the treadmill speed to accurately track a desired preset heart rate profile.

PID control is the most popular control algorithms in industry due to its simplicity in structure and ease of tuning. However, this study shows the acceptable heart rate tracking performance cannot be achieved by PID controller because of highly nonlinear behaviour of the controlled system. To achieve better tracking performance a new model based robust control strategy is developed, which successfully compensates the nonlinearity by using a Hammerstein model.

Fig. 1. A Hammerstein system.

The Hammerstein model can be described as a static nonlinear block (input nonlinearity) followed by a dynamic linear system (see Figure 2). Hammerstein models may account for nonlinear effects encountered not only in industrial processes [1] [2], but also in physiological processes [3] [4]. For example, electrically stimulated muscle [5], the upper limb [3], the muscle 
force [6], and the skeletal joints movements [7] can be effectively modelled by a nonlinear static element cascaded by a linear dynamic element.

To obtain the desired heart rate tracking result, this paper presents an integrated modelling and control approach based on Hammerstein models. This approach includes two integrated parts: the identification of Hammerstein model and model based robust control.

The identification of Hammerstein model is a very active research topic [8] [10] [14]. As suggested by Bai [15], there are mainly four kinds of approaches: 1) overparameterization method (two-stage procedures) [10] [16] [17]; 2) stochastic method (gray box procedure) [8] [18] [19]; 3) iterative method [14] [20]; 4) separable least squares method [21]. As discussed in [15], these approaches all have their own advantages and disadvantages. Recently, Goethals et al. [10] presented a novel overparametrization (two-stage procedures [22]) identification approach for Hammerstein systems. The most distinguishing part of that approach is the utility of a powerful machine learning method, Least Square Support Vector Machine (LSSVM) [23]. This novel machine learning method sets that paper apart from existing papers.

Support Vector Machine based regression [24] (Support Vector Regression (SVR)) is a new technique, which has been successfully applied to nonlinear function estimation [10] [23] [25]. Vapnik et al. established and developed the foundation of SVM [26] [27]. The formulation of SVM embodies the structure of the risk minimization principle, which has been shown to be superior to other traditional empirical risk minimization principles [28]. Support Vector Machine based regression applies the kernel methods implicitly to transform data into a feature space (this is known as a kernel trick [29]), and uses linear regression to get a non-linear function approximation in the feature space. SVR is very efficient in terms of speed and complexity, and successfully solves the over-fitting problem [30] by introducing regularization techniques.

This paper applies the SVM approach combined with the stochastic method [8] to identify physiological processes. There are at least two aspects which are different with respect to paper [10].

Firstly, the stochastic method [17] is employed in preference to the over parameterization method [8]. As discussed in [8] and [31], the error of the identification of Hammerstein model is not only from linear and nonlinear parts themselves but also from the coupling between them. The pseudo-random binary sequences (PRBS) are applied to decouple the identification of the two parts as suggested in [8]. 
Another main difference is the usage of $\epsilon$-insensitivity SVM [32] [33] instead of LS-SVM [23] [34] [35]. Both LS-SVM and $\epsilon$-insensitivity SVM have the merits of SVM approaches. However, the loss function used by $\epsilon$-insensitivity SVM, only penalizes errors greater than a threshold $\epsilon$ (See equation (3)). This leads to a sparse representation of the decision rule giving significant algorithmic and representation advantages [32]. On the other hand, the ridge regression $(\epsilon=0)$ used by LS-SVM typically often causes the loss of sparseness representation.

Most existing control methods for Hammerstein systems are based on direct inversion of the static nonlinearity combined with existing linear control approaches [2] [9]. These methods consider control and identification separately. They identify the Hammerstein model first and then invert the static nonlinearity part to obtain an approximated linear model. There are mainly two disadvantages of these approaches. Firstly, if the identified nonlinearity $\hat{f}(\cdot)$ has input multiplicity then the inversion is unachievable. Secondly, even when the inversion exists uniquely it is often hard to achieve an explicit analytic expression.

Based on $\epsilon$-insensitivity SVR, the inverse of the static nonlinearity rather than the nonlinearity is identified directly. Then, the $H_{\infty}$ control is designed for the approximated linear model to achieve robust tracking performance [36]. It should be noted the inverse of the static nonlinearity cannot be achieved directly by using the method proposed in [10].

In recent years, $H_{\infty}$ control has proved to be a very efficient tool for robust control design [37] [38] [39] [40]. $H_{\infty}$ control techniques have already been successfully applied in some biomedical problems (e.g. in automatic insulin injection for blood glucose regulation in diabetic patients [41].)

The proposed identification and $H_{\infty}$ based control approach was applied for the automated heart rate regulation system design, and excellent tracking results are achieved. This is also the first report of an SVM application in the area of cardiovascular system identification and control practice.

The paper is organized as follows. The details of SVM based identification and control approach are given in Section II. Section III presents the application of the proposed approach for the regulation of heart rate of treadmill exerciser. 


\section{SVM BASED IDENTIFICATION AND CONTROL APPROACH}

In this paper, we use a Hammerstein model to dynamically describe the relationship between walking speed and heart rate variation. We do this for the following reasons. The steady state relationship between walking speed and work load is nonlinear. The dynamic linearity between work load (in the range from 50 to 125 Watt) and heart rate variation was established by, for example, Hajek et al. [42]. It is therefore reasonable to use a nonlinear static element cascaded by a linear dynamic element to approximately describe the relationship during moderate treadmill exercises.

In this study, as assumed in most gray box procedure (or stochastic method) [8], the steadystate gain of the linear dynamic model is constrained to be unity, and the steady-state characteristic of the overall model is determined by the static nonlinearity. As mentioned in the introduction, the linear dynamic identification of Hammerstein models can be decoupled from that of nonlinear parts by using pseudo-random binary sequences [8]. However, the PRBS inputs often cannot excite the nonlinearity sufficiently. To identify the nonlinear part or its inverse,

steady state experiments should be performed. In this section the identification of the inverse of nonlinear part is introduced first.

A. Modelling the inverse of the nonlinear function by using SVR

Fig. 2. The precompensated Hammerstein system.

To transfer a Hammerstein system to a linear system, a pre-compensator as shown in Fig. 3 can be applied as in [9].

For the identification of the inverse of nonlinearity, the so called $\epsilon$-insensitivity SVR regression will be employed, which is convex and very efficient in terms of speed and complexity.

Now we briefly introduce the SVR approach [43]. 
Let $\left\{u_{i}, y_{i}\right\}_{i=1}^{N}$ be a set of inputs and outputs data points $\left(u_{i} \in U \subseteq \mathcal{R}^{d}, y_{i} \in Y \subseteq \mathcal{R}, N\right.$ is the number of points). The goal of the support vector regression is to find a function $f(u)$ which has the following form

$$
f(u)=w \cdot \phi(u)+b,
$$

where $\phi(u)$ represents the high-dimensional feature spaces which are nonlinearly transformed from $u$. The coefficients $w$ and $b$ are estimated by minimizing the regularized risk function:

$$
\frac{1}{2}\|w\|^{2}+C \frac{1}{N} \sum_{i=1}^{N} L_{\epsilon}\left(y_{i}, f\left(u_{i}\right)\right)
$$

The first term is called the regularized term. The second term is the empirical error measured by $\epsilon$-insensitivity loss function which is defined as:

$$
L_{\epsilon}\left(y_{i}, f\left(u_{i}\right)\right)=\left\{\begin{array}{cl}
\left|y_{i}-f\left(u_{i}\right)\right|-\epsilon, & \left|y_{i}-f\left(u_{i}\right)\right|>\epsilon \\
0, & \left|y_{i}-f\left(u_{i}\right)\right| \leq \epsilon
\end{array}\right.
$$

This defines an $\epsilon$ tube. The radius $\epsilon$ of the tube and the regularization constant $C$ are both determined by user.

The selection of parameter $C$ depends on application knowledge of the domain. Theoretically, a small value of $C$ will under-fit the training data because the weight placed on the training data is too small, thus resulting in large values of MSE (mean square error) on the test sets. However, when $C$ is too large, SVR will over-fit the training set so that $\frac{1}{2}\|\omega\|$ will lose its meaning and the objective goes back to minimizing the empirical risk only. Parameter $\epsilon$ controls the width of the $\epsilon$-insensitive zone. Generally, the larger the $\epsilon$ the fewer number of support vectors and thus the sparser the representation of the solution. However, if the $\epsilon$ is too large, it can deteriorate the accuracy on the training data.

By solving the above constrained optimization problem, we have

$$
f(u)=\sum_{i=1}^{N} \beta_{i} \phi\left(u_{i}\right) \cdot \phi(u)+b .
$$

As mentioned before, by the use of kernels, all necessary computations can be performed directly in the input space, without having to compute the map $\phi(u)$ explicitly. After introducing kernel function $k\left(u_{i}, u_{j}\right)$, the above equation can be rewritten as follows.

$$
f(u)=\sum_{i=1}^{N} \beta_{i} k\left(u_{i}, u\right)+b .
$$


Where the coefficients $\beta_{i}$ corresponding to each $\left(u_{i}, y_{i}\right)$. The support vectors are the input vectors $u_{j}$ whose corresponding coefficients $\beta_{j} \neq 0$.

For linear support regression, the kernel function is thus the inner product in the input space:

$$
f(u)=\sum_{i=1}^{N} \beta_{i}<u_{i}, u>+b .
$$

For nonlinear SVR, there are a number of kernel functions which have been found to provide good generalization capabilities, such as polynomials, radial basis function (RBF), sigmod. Here we give the polynomials and RBF kernel functions as follows:

RBF kernel: $k\left(u, u^{\prime}\right)=\exp \left(-\frac{\left\|u-u^{\prime}\right\|^{2}}{2 \sigma^{2}}\right)$,

Polynomial kernel: $k\left(u, u^{\prime}\right)=\left(\left(u \cdot u^{\prime}\right)+h\right)^{p}$.

Details about SVR, such as the selection of radius $\epsilon$ of the tube, kernel function, and the regularization constant $C$, can be found in [29] [43].

It should be emphasized that, as we need to model the inverse of the nonlinear function $f$ (see Fig. 3), the measured steady state output $y$ (heart rate) will be used as the input data, and the input $\tilde{u}$ (treadmill speed) as the output data.

\section{B. Identification of linear dynamic part}

In [8], Bai showed that the identification of linear part of a Hammerstein model can be decoupled from nonlinear part with the help of the PRBS input. The reason is that any static nonlinearity can be exactly characterized by a linear function, when driven by PRBS inputs which have a binary nature.

When a PRBS input is employed for the identification of the Hammerstein system, as shown in equation (2.3) of [8], the identification of a Hammerstein model can be simplified as a linear identification problem. Furthermore, the correlation analysis method can be applied to identify impulse responses the linear dynamic part. 


\section{Model predictive controller design}

Fig. 3. A typical Model predictive controller.

After the pre-compensator is employed, the Hammerstein system can be treated as a linear dynamic system. In [], we presented a $H_{i} n f t y$ based control approach for the control of heart rate response with exercises and obtained desired tracking performance for healty young exercisers. In this study, we plan to cope with heart rate regulating for old exercisiers. In order to ensure the safety of old exercisers, the strength (walking speed) of treadmill exercises must be restricted to a moderate level. Furthermore, as the delayed response of treadmill and cardivascular system of old exercises, the model predictive control is the most suitable selection.

In this study, the input disturbances and additive model uncertainty are considered as modelling errors. In order to achieve desired tracking performance under input disturbance and additive model uncertainty, an $H_{\infty}$ controller is employed for the pre-compensated Hammerstein system. This controller design problem can be formulated as a mixed sensitivity problem [36] [37] [44] [45] [46] as shown in Fig. 4. $P$ is the process to be controlled, and $K$ is the robust controller. $w_{d}$ is the exogenous input (disturbance or reference input). $Z_{1}$ and $Z_{2}$ are the controlled variables. $W_{Z_{1}}$ and $W_{Z_{2}}$ are the complementary sensitivity weighting function and sensitivity weighting function respectively. The formulated mixed sensitivity problem is the design of the robust controller $K$ to ensure that the weighted sensitivity function and complementary sensitivity function are bounded so that both robust performance and the desired stability margin can be obtained [37] [47] [48].

General guidelines for weighting function selection can be found in, for example [49] [44]. In order to obtain offset free tracking, a commonly used method is to include an integrator in the sensitivity weighting function $W_{Z_{2}}$. If model uncertainty $\Delta(j \omega)$ is known, we normally need to select the complementary sensitivity weighting function $W_{Z_{1}}$ such that $\left|W_{Z_{1}}(j \omega)\right|>$ $\bar{\sigma}(\Delta(j \omega))$ for all $\omega$ to obtain robust stability. However, deriving the model uncertainty $\Delta(j \omega)$ 
is very challenging especially for nonlinear systems. In this study, we firstly select $W_{Z_{1}}$ such

that $\left|W_{Z_{1}}(j \omega)\right|$ is big enough to ensure robust stability. Then, gradually decrease $\left|W_{Z_{1}}(j \omega)\right|$ to diminish the conservativeness.

\section{Heart RATE Regulation APPLication}

The major aim of this section is to apply the proposed identification and control approach to develop a computer controlled treadmill system, which can automatically control the treadmill speed to accurately track a desired preset heart rate profile.

\section{A. Experimental equipment}

The computer controlled treadmill and its related data collection and processing system are shown in Fig. 5 and 6.

Fig. 4. The computer controlled treadmill system.

Fig. 5. Block diagram for computer controlled treadmill system.

The treadmill used in the system is the Powerjog "G" Series fully motorized medical grade treadmill manufactured by Sport Engineering Limited, England. Control of the treadmill can be achieved through an RS232 serial port. The treadmill can receive commands from the computer 
controller via this link, and obeys such commands without supervision. The measurement of heart rate in the designed system is implemented using a wireless Polar system. However, even in the absence of external interference the heart rate can vary substantially over time under the influence of various internal or external factors. Therefore, an improved exponential weighted moving average filter together with a simple outlier detection algorithm [13] [50] [51] is adopted for the estimation of the heart rate. Specifically, the control computer collects heart rate signal from a Polar receiver through an analog input port every 2 seconds, and calculates heart rate by using an edge detection algorithm. Only measured heart rate within a reasonable range (for example, between 50 and 150 beats per minute) was counted in the measured sequence in order to remove outliers.

Assume $\left\{x_{k}\right\}$ is the sequence of the measured heart rate. We apply the exponentially weighted moving average filter to the sequence $\left\{x_{k}\right\}$ :

$$
\bar{x}_{k}=\alpha \bar{x}_{k-1}+(1-\alpha) x_{k-1} .
$$

where $\alpha$ is the filter coefficient. In this study, the value of $\alpha$ was selected as 0.35 , to achieve optimal filtering effects.

\section{B. Nonlinearity modelling by using Support Vector Regression}

In order to identify the nonlinear relationship, steady state experiments are performed and recorded. Six young healthy male subjects volunteered to participate in the study. Their physical characteristics are presented in Table I.

\section{TABLE I. Subject characteristics ( $\mathbf{N}=\mathbf{6})$.}

All experiments were conducted in the afternoon, and the subjects were permitted to have a light meal one hour before experiments. Initially, the subjects were asked to walk for about 
10 minutes on the treadmill to familiarize themselves with the experiment. The subjects were then requested to walk at five levels of different speeds $(3 \mathrm{~km} / \mathrm{h}, 4 \mathrm{~km} / \mathrm{h}, 5 \mathrm{~km} / \mathrm{h}, 6 \mathrm{~km} / \mathrm{h}$ and a subject specific maximum walking speed, typically $7 \mathrm{~km} /$ hour). Each level took a total period of 5 minutes, and was followed by a 10-minute resting period. Finally, in order to identify the linear dynamic part of the Hammerstein system, subjects were also requested to walk on the treadmill under a PRBS input as shown in Fig. 7. The detailed descriptions of this PRBS signal is given in Subsection III.C. Throughout the experiments heart rates were recorded.

In this paper, both linear and $\epsilon$-insensitivity SVR regression methods are applied to model the inverse of the nonlinear function $(f)$. We randomly select 22 data points (73\% of the total data points) to train the model, and 8 data points (27\% of total data points) to test the regression results. Comparing SVR regression with linear regression, the former obtains the better results. SVR regression decreases the training error (Mean Square Error) from $0.294(\mathrm{~km} / \mathrm{h})^{2}$ to 0.259 $(\mathrm{km} / \mathrm{h})^{2}$, while testing error decreases from $0.382(\mathrm{~km} / \mathrm{h})^{2}$ to $0.265(\mathrm{~km} / \mathrm{h})^{2}$. This partially proves the steady state relationship between walking speed and heart rate is nonlinear. After such comparisons, we utilize all experimental data to perform SVR regression in order to include as much as experimental information in the model as possible. The detailed regression results, such as the selected design parameters (including insensitivity region $\epsilon$, kernel function, and the regularization constant $C$ ) and the support vector number, are summarized in Table II and Fig. 8. In Fig. 8, the continuous curve stands for the estimated input output steady state relationship. The dotted lines indicate the $\epsilon$-insensitivity tube. The plus markers are the points of input and output data. The circled plus markers are the support points. The quality of control (see Subsection III.E) also demonstrates that this nonlinear SVR model is a good representation of the inverse of the nonlinear function. It should be emphasized that $\epsilon$-insensitive SVR just uses 5 points (16.7 $\%$ of the total points) to sparsely describe the nonlinear relationship efficiently. 
TABLE. II. Details about the estimation of the inverse of the nonlinear function by SVR

Fig. 6. Two periods of 31 bits PRBS inputs (treadmill speed) and its corresponding outputs (averaged heart rate of six subjects).

\section{Linear dynamic modelling}

From a physiological analysis of cardiovascular systems [52], two transitory components are known to contribute to the heart rate response: at the beginning of exercise the rapid component of vagal inhibition and then a much slower acting complex of sympathetic effects. For the dynamic modelling of heart rate variation during exercise, some complicated model structures [53] [42] are proposed based on physiological analysis. However, from a control application point of view, these models are too complicated and often lead to poor determinability of parameters and thus poor control performance. Hajek et al. proposed a simpler version of the model presented in [42] as follows:

$$
Y(s)=\left[\frac{K_{1} T_{i} s}{\left(T_{1} s+1\right)\left(T_{i} s+1\right)} \quad \frac{K_{r e f}}{T_{i} s+1}\right]\left[\begin{array}{c}
U_{1}(s) \\
U_{2}(s)
\end{array}\right]
$$

In equation (7), $Y(s)$ is the Laplace form of the heart rate variation $y(t)$, and input $u_{2}(t)$ ( $U_{2}(s)$ is its Laplace form) is the workload. Workload is the amount of power being demanded of an exercising subject, manifesting itself to the subject as exercise intensity. Input $u_{1}(t)\left(U_{1}(s)\right.$ is its Laplace form) is constant 1 when $u_{2}(t)>0$, zero when $u_{2}(t)=0$. As input $u_{1}(t)$ is dependent on $u_{2}(t)$, it is therefore not an independent input. Furthermore, the fast component (which is associated with $u_{1}(t)$ ) cannot be observed distinctly in our experimental data. Thus, the effects of $u_{1}(t)$ is neglected but treated as model uncertainty in order to reduce design complexity. Therefore, a first order model (with input disturbances) to model the heart variation 
during exercise is a good choice from physiological analysis. Later, we will show this model structure is also confirmed by two popular model selection criteria.

In this study, a PRBS [54] [55] input is employed to decouple the identification of linear dynamic from nonlinearity of the Hammerstein system. A PRBS input (see Fig. 7) has two levels $\left(u(t)=u_{1}\right.$ or $\left.u_{2}\right)$ and may switch from one level to the other only at constant time intervals $T_{s}$. A PRBS is periodic with period $T=T_{s} N$, where $\mathrm{N}$ is an integer. In this study, $u_{1}=4 \mathrm{~km} / \mathrm{h}, u_{2}=6 \mathrm{~km} / \mathrm{h}, N=31$, and $T_{s}=15 \mathrm{~s}$. An important issue for the PRBS based experiment implementation is the synchronization of the input PRBS signal and the output measurement. In this study, the triaxial accelerometers are mounted on the lower back of treadmill exerciser to synchronize body movements with measured ECG signal, which ensures reliable estimation of time delay (See Figure 9).

Fig. 7. Modelling of the inverse of the nonlinear function by using $\epsilon$-insensitivity SVR.

Fig. 8. The triaxial accelerometer monitored two periods of 31 bits PRBS signal.

Based on experimental data (see Figure 7), the structure is determined by using the Matlab Identification Toolbox. Two popular model selection criteria Akaike information criterion (AIC) and minimum description length (MDL) were used to select the first order ARX (Auto Regressive eXternal) model with time delay as the best model structure for linear dynamics. The identified 
linear model is given as follows:

$$
y(k)=0.648 y(k-1)+0.352 u(k-3)+e(k),
$$

with sampling period $T_{s}=15$ seconds. Note the time delay contained in equation (8) includes the delay of the response of human cardiovascular system as well as the lag time of the automated treadmill system.

\section{Robust tracking controller design}

The identified model inevitably has modelling errors. In this study, the modelling errors are considered in two forms: input disturbance and model uncertainty. To achieve robust tracking under input disturbances and model uncertainty, a mixed sensitivity $H_{\infty}$ controller [37] [45] [46] is designed for the identified model. As the design of discrete time mixed sensitivity $H_{\infty}$ controllers is not as mature as for continuous time systems, the design of the weighting function of the mixed sensitivity $H_{\infty}$ problem is implemented by using its continuous counterpart. Specifically, we convert the discrete time model, equation (8) (with sampling period $T_{s}=15$ seconds) into a continuous time model by using the matched pole-zero method of [56]. The weighting function in this study is recursively selected based on general guidelines of [49] [44]. In order to obtain offset free tracking, we select the sensitivity weighting function in the form of $W_{Z_{2}}=\frac{k_{z_{2}}}{(s+\alpha)}$ (with $\alpha$ sufficiently small). We select the complementary sensitivity weighting function as $W_{Z_{1}}=\frac{s}{k_{z_{1}}}$ to acquire desired stability margin as well as to satisfy rank condition (full rank requirement of matrix $D_{21}$ of the augmented process) of standard $H_{\infty}$ problem. After several recursive design steps, we obtained the following set of parameters for the weighting functions to obtain an augmented model for the weighted process: $k_{z_{1}}=1000, k_{z_{2}}=0.018$, and $\alpha=0.0001$.

The augmented continuous model is then converted to its discrete time form. Finally, a mixed sensitivity $H_{\infty}$ controller can be designed for this discrete time model:

$$
K(z)=\frac{0.3487-0.6466 z-0.2899 z^{2}+0.6279 z^{3}}{-0.3779+1.174 z-1.79 z^{2}+z^{3}} .
$$


E. Heart rate regulation results

Fig. 9. Typical step response comparison between proposed approach and conventional PID control.

Fig. 10. Step responses for all subjects.

The step responses of heart rate control by using PID and the proposed control approach are compared in Fig. 10. The step responses for all subjects are shown in Fig. 11. It can be seen that the proposed controller achieved much better performance than conventional PID control. The main reason is the compensation of the system nonlinearity by using a Hammerstein model for the heart rate. Specifically, the heart rate variation due to a change of walking speed from a slow speed (for example, from $3 \mathrm{~km} / \mathrm{h}$ to $3.5 \mathrm{~km} / \mathrm{h}$ ) is much smaller than that for the same alternation from a fast speed (for example, from $6 \mathrm{~km} / \mathrm{h}$ to $6.5 \mathrm{~km} / \mathrm{h}$ ). The proposed approach copes with such difference by using the identified inverse of the nonlinear function $(f)$ as a precompensator (see Fig. 3) to systematically compensate for the nonlinearity, whereas linear controllers (such as conventional PID controllers) cannot.

Finally, the proposed controller was used to track a preset desired heart rate profile. The profile includes three stages: 5 minutes increasing (from resting heart rate to $110 \mathrm{beats} / \mathrm{min}$ ), 15 minutes holding (110 beats/min), and 5 minutes decreasing (from 110 beats/min to normal.). This 
is comparable to heart rate profiles recommended for optimal low level aerobic training at about $60 \%$ of maximum effort. Figures 12 and 13 below demonstrate that quite satisfactory tracking of the desired heart rate profile was achieved. From the shape of treadmill speed shown in figures 12 and 13, it is also observed that the treadmill speed is decreased in a slightly different rate for different subjects whilst the heart rate is maintained at around $110 \mathrm{bpm}$. These data suggest the hypothesis that fit subjects will have a low rate of decrease of treadmill speed. This hypothesis is being further tested in our laboratory, using the newly designed computer controlled treadmill.

Fig. 11. A typical heart rate tracking for an exercising heart rate profile.

Fig. 12. Heart rate tracking results for all subjects.

\section{CONCLUSION}

In this paper, an integrated modelling and control approach is developed for Hammerstein systems as applied to the regulation of cardiovascular system response to exercise. The stochastic method is adopted to decouple the identification of linear and nonlinear parts. Powerful $\epsilon$ insensitivity Support Vector Regression is employed to identify the inverse of input nonlinearity in order to transfer Hammerstein systems to linear systems. A robust $H_{\infty}$ tracking controller is then designed for the converted Hammerstein system. The approach is successfully applied to a 
heart rate regulation system for exercise on a motorized treadmill. This is the first report of an SVM application in the cardiovascular system identification and control practice, and achieves excellent results. It should be mentioned that because of the sparse representation [32] [57] of the static nonlinearity obtained by using $\epsilon$-insensitivity SVR, the implementation complexity is greatly reduced. We believe that the ability to track a predetermined heart rate profile may be useful in cardiac rehabilitation programs or for safer exercise for individuals at risk. 


\section{REFERENCES}

[1] E. Eskinat, S. Johnson, and W. Luyben, "Use of Hammerstein models in identification of nonlinear systems," AIChE J., vol. 37, pp. 255-268, 1991.

[2] S. Su, J. Bao, and P. Lee, "Control of multivariable Hammerstein systems by using feedforward passivation,” Ind. \& Eng. Chem. Res., no. 4, pp. 891-899, 2005.

[3] J. Allin and G. Inbar, "Fns parameter selection and upper limb characterization," IEEE Trans. Biomed. Eng., pp. 809-817, 1986.

[4] K. Hunt, M. Munih, N. Donaldson, and F. Barr, "Investigation of the Hammerstein hypothesis in the modeling of electrically stimulated muscle,” IEEE Trans. Biomed. Eng., no. 8, pp. 998-1009, 1998.

[5] L. Bernotas, P. Crago, and H. Chizeck, "A discrete-time model of electrically stimulated muscle," IEEE Trans. Biomed. Eng., pp. 829-838, 1986.

[6] G. Wilhere, P. Crago, and H. Chizeck, "Design and evaluation of a digital closed-loop controller for the regulation of muscle force by recruitment modulation,” IEEE Trans. Biomed. Eng., pp. 668-676, 1985.

[7] L. Vodovnik, W. Crochetiere, and J. Reswick, "Control of skeletal joints by electrical stimulation," Med. Biol. Eng., pp. 97-109, 1967.

[8] E. Bai, "Decoupling the linear and nonlinear parts in Hammerstein model identification," Automatica, vol. 40, pp. 671-676, 2004.

[9] G. Ingram, M. Franchek, V. Balakrishnan, and G. Surnilla, "Robust SISO $\mathcal{H}_{\infty}$ controller design for nonlinear systems," Control Engineering Practice, vol. 13, pp. 1413-1423, 2005.

[10] I. Goethals., K. Pelckmans, J. Suykens, and B. De Moor, "Identification of mimo Hammerstein models using least squares support vector machines," Automatica, vol. 41, pp. 1263-1272, 2005.

[11] R. Cooper, S. Horvath, J. Bedi, D. Drechsler-Parks, and R. Williams, "Maximal exercise responses of paraplegic wheelchair road racers," Paraplegia, vol. 30, pp. 573-581, 1992.

[12] H. Lakomy, I. Cambell, and C. Williams, "Treadmill performance and selected physiological characteristics of wheelchair athletes," British J. Sports Medicine, vol. 21, no. 3, pp. 130-133, 1987.

[13] S. Su, L. Wang, B. Celler, and A. Savkin, "Heart rate control during treadmill exercise," in Proceedings of the 27th Annual International Conference of the IEEE Engineering in Medicine and Biology Society (EMBS), pp. 2471-2474, September, 2005, Shanghai, China.

[14] K. Narendra and P. Gallman, "An iterative method for the identification of nonlinear systems using Hammerstein model," IEEE Transactions on Automatic Control, vol. 11, pp. 546-550, 1966.

[15] E. Bai, "A blind approach to the Hammerstein model identification," IEEE Tran. on Signal Processing, no. 7, pp. 16101619, 2002.

[16] F. Chang and R. Luus, "A noniterative method for identification using Hammerstein model," IEEE Trans. Automat. Contr., pp. 464-468, 1971.

[17] E. Bai, "An optimal two-stage identification algorithm for Hammerstein- wiener nonlinear systems," Automatica, no. 3, pp. 333-338, 1998.

[18] S. Bilings and S. Fakhouri, "Identification of a class of nonlinear systems using correlation analysis," Proc. Inst. Elect. Eng., no. 7, pp. 691-697, 1978.

[19] M. Pawlak, "On the series expansion approach to the identification of Hammerstein systems," IEEE Trans. Automat. Contr., pp. 763-767, 1991. 
[20] J. Voros, "Parameter identification of discontinuous Hammerstein systems," Automatica, no. 6, pp. 1141-1146, 1997.

[21] A. Ruhe and P.Wedin, "Algorithms for separable nonlinear least squares problems," SIAM Rev., pp. 318-337, 1980.

[22] P. Falugi, L. Giarre, and G. Zappa, "Approximation of the feasible parameter set in worst-case identification of Hammerstein models," Automatica, vol. 41, pp. 1017-1024, 2005.

[23] J. Suykens, Van Gestel, J. De Brabanter, B. De Moor, and J. Vandewalle, Least squares support vector machines. Singapore: WorldScientific, 2002.

[24] H. Drucker, C. Burges, L.Kaufman, A. Smola, and V. Vapnik, "Support vector regression machines," in Advances in Neural Information Procession Systems, M. Mozer, M. Jordan, and T. Petsche, Eds. Cambridge, MA, 1997, pp. 155-161.

[25] S. Su, L. Wang, B. Celler, E. Ambikairajah, and A. Savkin, "Estimation of walking energy expenditure by using support vector regression," in Proceedings of the 27th Annual International Conference of the IEEE Engineering in Medicine and Biology Society (EMBS), pp. 3526-3529, September, 2005, Shanghai, China.

[26] V. Vapnik, The Nature of Statistical Learning Theory. New York: Springer, 1995.

[27] V. Vapnik and A. Lerner, "Pattern recognition using generalized portrait method," Automation and Remote Control, 1963.

[28] S. Gunn, M. Brown, and K. Bossley, "Network performance assessment for neuro-fuzzy data modelling," Intelligent Data Analysis, pp. 313-323, 1997.

[29] B. Schlkopf and A. Smola, Learning with kernels. Cambridge: MA: MIT Pres, 2002.

[30] Y. Guo, P. Bartlett, J. Shawe-Taylor, and R. Williamson, “Covering numbers for support vector machines,” IEEE Trans. on Information Theory, no. 1, pp. 239-250, 2002.

[31] B. Ninness and S. Gibson, "Quantifying the accuracy of Hammerstein model estimation,” Automatica, vol. 38, pp. 20372051, 2002.

[32] N. Cristianini and J. Shawe-Taylor, An Introduction to Support Vector Machines and other kernel-based learning methods. UK: Cambridge University Press, 2000.

[33] A. Smola and B. Schlkopf, "A tutorial on support vector regression," Statistics and Computing, pp. 199-222, 2004.

[34] M. Espinoza, K. Pelckmans, L. Hoegaerts, J. Suykens, J. De Brabanter, and B. De Moor, “A comparative study of 1s-svms applied to the silver box identi.cation problem," Symposium on nonlinear control systems NOLCOS, pp. 513-518, 2004.

[35] J. Suykens, Van Gestel, J. De Brabanter, B. De Moor, and J. Vandewalle, "Least squares support vector machines," Neurocomputing, vol. 48, pp. 85-105, 2002.

[36] S. Su, B. Anderson, and T. Brinsmead, "Robust disturbance suppression for nonlinear systems based on $\mathcal{H}_{\infty}$ control," Proceedings of the 39th Conference on Decision and Control, pp. 3013-3018, 2000.

[37] K. Zhou, J. C. Doyle, and K. Glover, Robust and Optimal Control. New Jersey: Prentice-Hall, 1996.

[38] A. Savkin and I. Petersen, "A connection between $\mathcal{H}_{\infty}$ control and absolute stabilizability of uncertain systems," Systems and Control Letters, pp. 197-203, 1994.

[39] I. Petersen, V. Ugrinovskii, and A. Savkin, Robust Control Design Using $\mathcal{H}_{\infty}$ Methods. London: Springer-Verlag, 2000.

[40] A. Savkin and I. Petersen, "Nonlinear versus linear control in the absolute stabilizability of uncertain linear systems with structured uncertainty," IEEE Transactions on Automatic Control, no. 1, pp. 122-127, 1995.

[41] F. Chee, A. Savkin, T. Fernando, and S. Nahavandi, "Optimal $\mathcal{H}_{\infty}$ insulin injection control for blood glucode regulation in diabetic patients," IEEE Trans. on Biomedical Engineering, no. 10, pp. 1625-1631, 2005.

[42] M. Hajek, J. Potucek, and V. Brodan, "Mathematical model of heart rate regulation during exercise," Automatica, vol. 16, pp. 191-195, 1980.

[43] V. Vapnik, Statistical Learning Theory. New York: John Wiley, 1998. 
[44] T. Mita, M. Hirata, K. Murata, and H. Zhang, " $\mathcal{H}_{\infty}$ control versus disturbance-observer-based control," IEEE Transactions on Industrial Electronics, pp. 488-495, JUNE 1998.

[45] S. Su, B. Anderson, and T. Brinsmead, "Use of integrator in nonlinear $\mathcal{H}_{\infty}$ design for disturbance rejection," Automatica, vol. 38, no. 11, pp. 1951-1957, 2002.

[46] M. Green and D. J. N. Limebeer, Linear Robust Control. New Jersey: Prentice Hall, 1995.

[47] I. Petersen and A. Savkin, Robust Kalman Filtering for Signals and Systems with Large Uncertainties. Boston: Birkhauser, 1999.

[48] A. Savkin and I. Petersen, "state estimation and model validation for discrete- time uncertain systems with a deterministic description of noise and uncertainty," Automatica, no. 2, pp. 271-274, 1998.

[49] R. Chiang and M. Safonov., Robust Control Toolbox 2.0. The MathWorks, Inc, 1992.

[50] S. Su, L. Wang, B. Celler, A. Savkin, and Y. Guo, "Modelling and control for heart rate regulation during treadmill exercise," in Proceedings of the 28th Annual International Conference of the IEEE Engineering in Medicine and Biology Society (EMBS), September, 2006, New York, USA.

[51] S. Su, L. Wang, B. Celler, and A. Savkin, "Estimation of oxygen consumption for moderate exercises by using a hammerstein model," in Proceedings of the 28th Annual International Conference of the IEEE Engineering in Medicine and Biology Society (EMBS), September, 2006, New York, USA.

[52] C. Best and N. Taylor, The Physiological Basis of Medical Practice. London: Bailliera Tindal and Cox, 6th ed., 1955.

[53] V. Brodan, M. Hajek, and E. Khun, "An analog model of pulse rate during physical load and recovery," Physiol. Bohemoslov, vol. 20, no. 2, p. 189, 1971.

[54] P. Briggs, K. Godfrey, and P. Hammond, "Identification and process parameter estimation," IFAC Symp. on the Problems of Identification in Automatic Control Systems, 1967.

[55] W. Davies, System Identification for Self-Adaptive Control. New York: Wiley-Interscience, 1970.

[56] G. Franklin, J. Powell, and M. Workman, Digital Control of Dynamic Systems. Addison-Wesley, 1990.

[57] J. Suykens, J. De Brabanter, L. Lukas, and J. Vandewalle, "Weighted least squares support vector machines: Robustness and sparse approximation," Neurocomputing, vol. 48, pp. 85-105, 2002. 


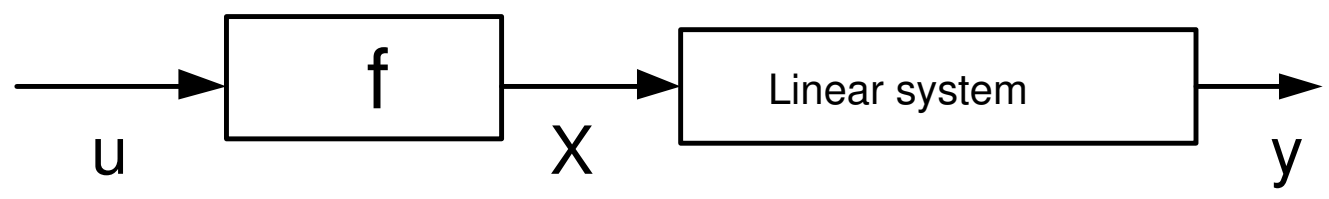

Fig. 2. A Hammerstein system. 


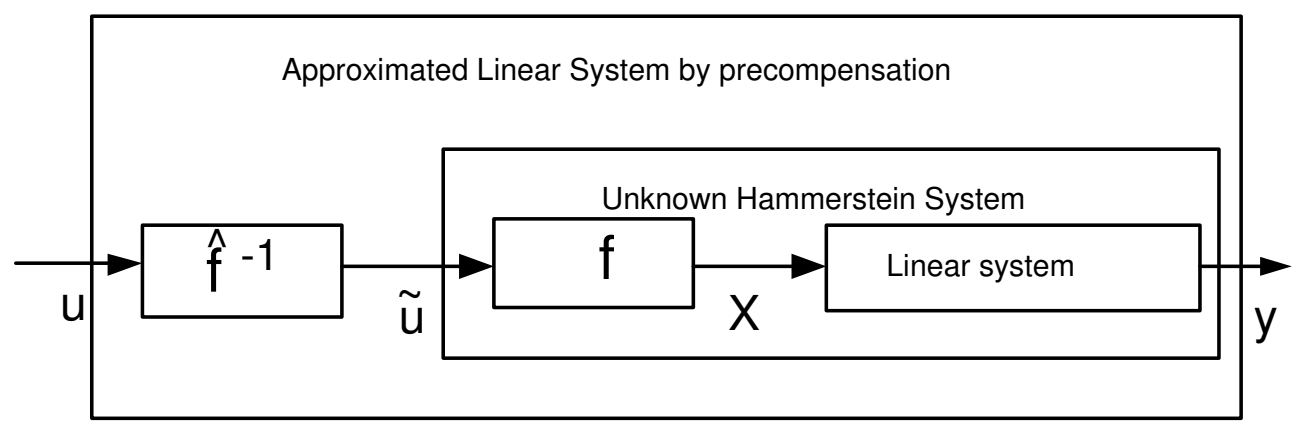

Fig. 3. The precompensated Hammerstein system 


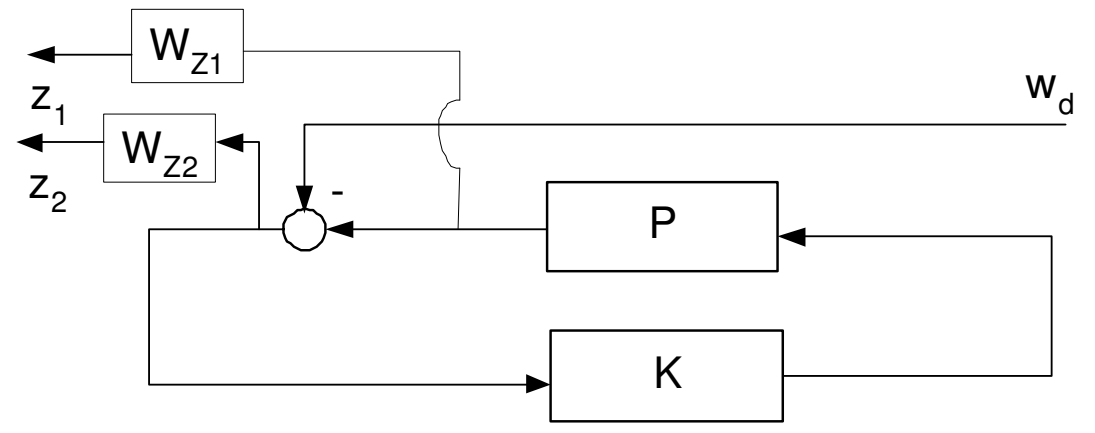

Fig. 4. A mixed sensitivity $H_{\infty}$ control problem 


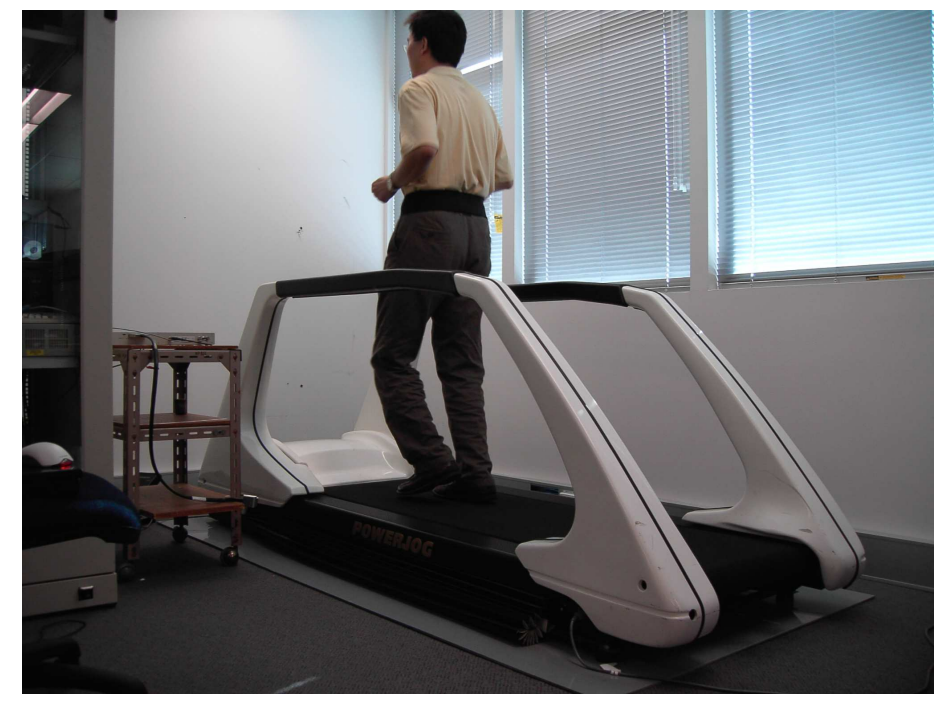

Fig. 5. The computer controlled treadmill system 


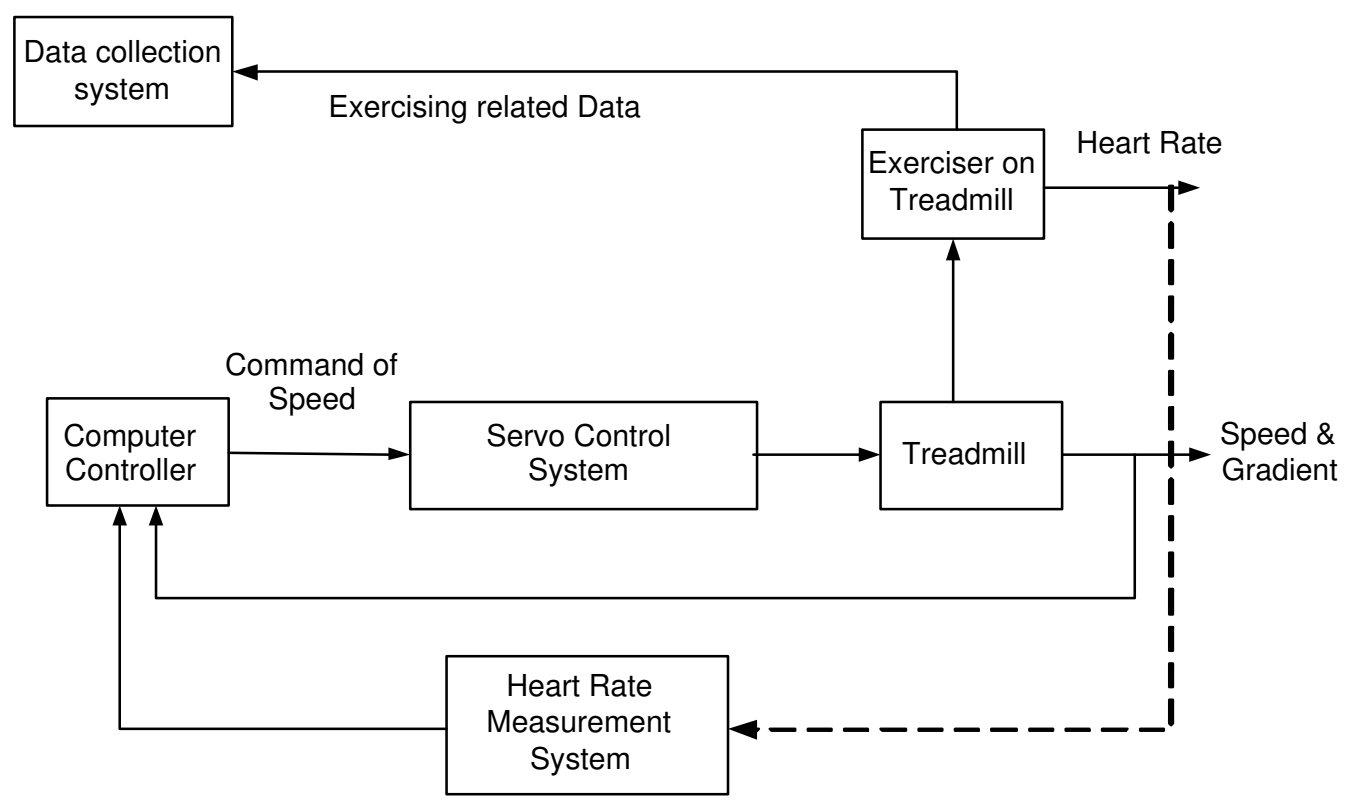

Fig. 6. Block diagram for computer controlled treadmill system 

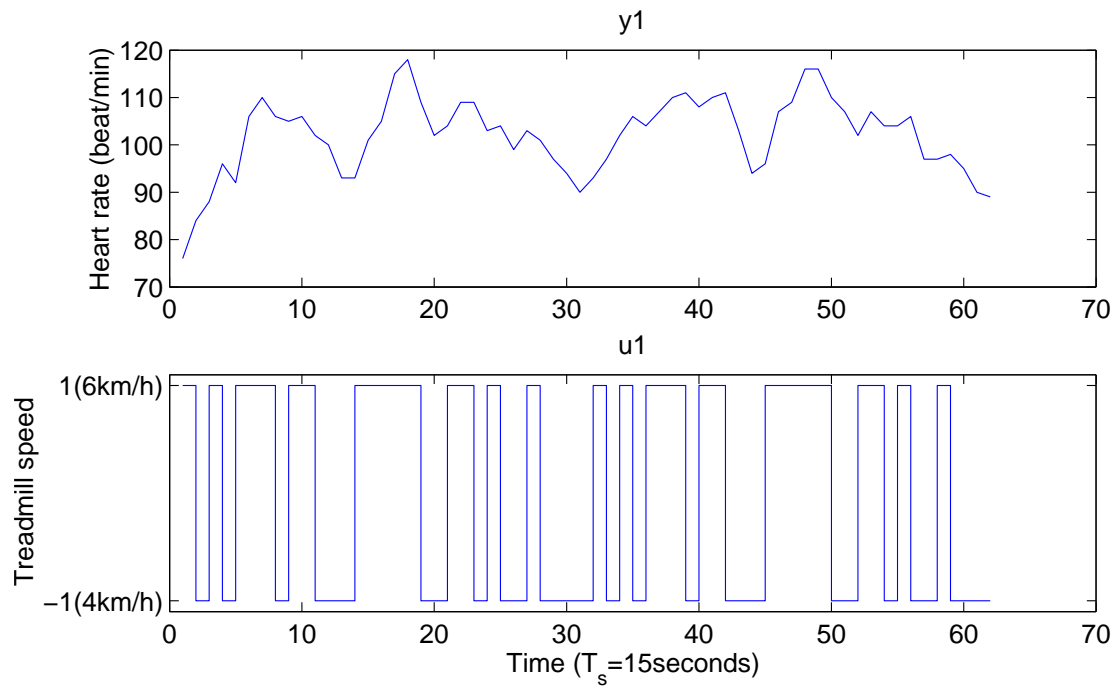

Fig. 7. Two periods of 31 bits PRBS inputs (treadmill speed) and its corresponding outputs (averaged heart rate of six subjects) 


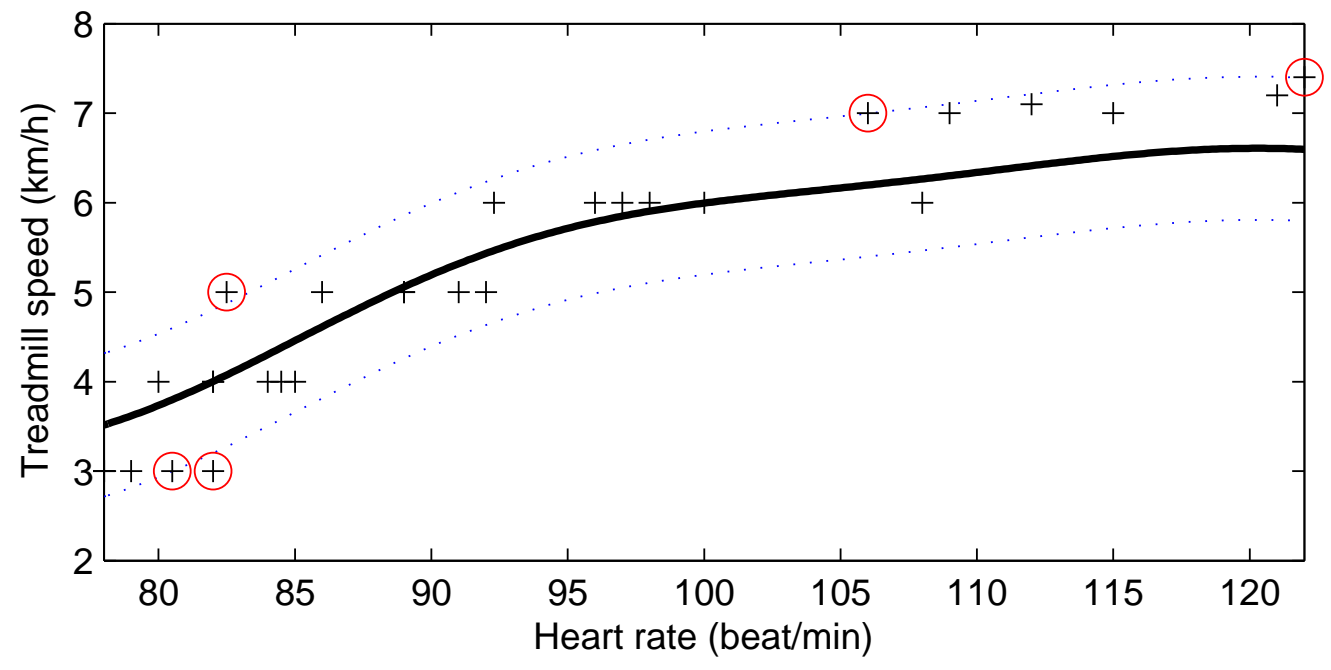

Fig. 8. Modelling of the inverse of the nonlinear function by using $\epsilon$-insensitivity SVR 

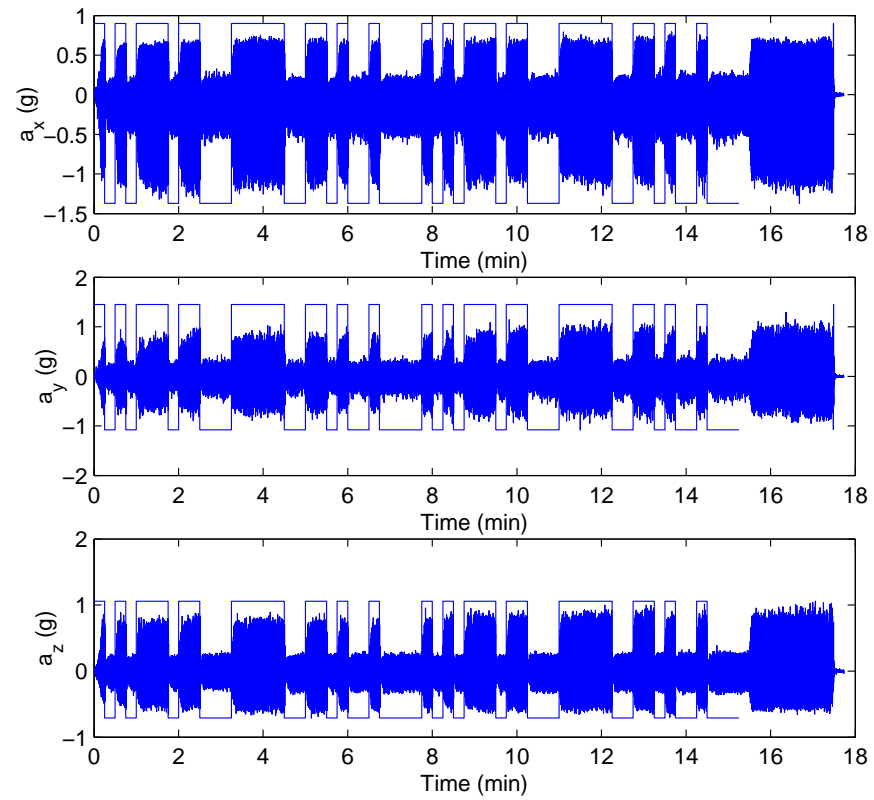

Fig. 9. The triaxial accelerometer monitored two periods of 31 bits PRBS signal 

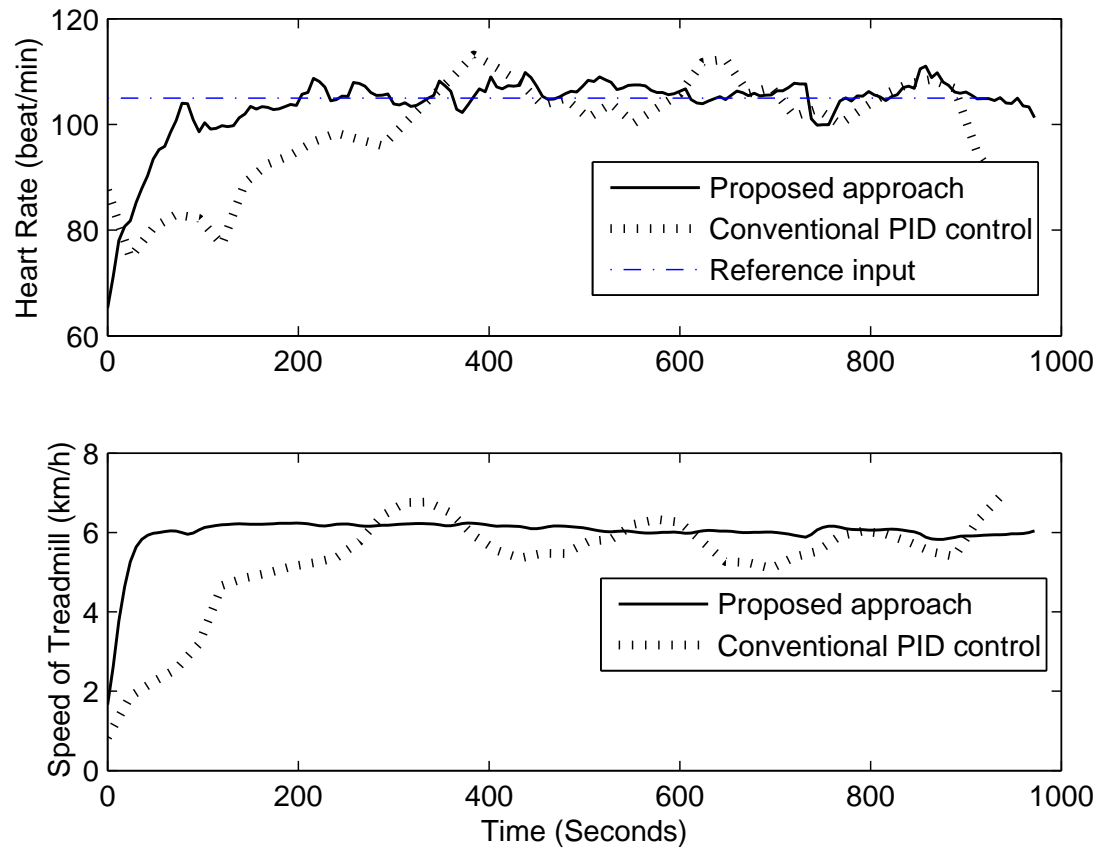

Fig. 10. Typical step response comparison between proposed approach and conventional PID control 

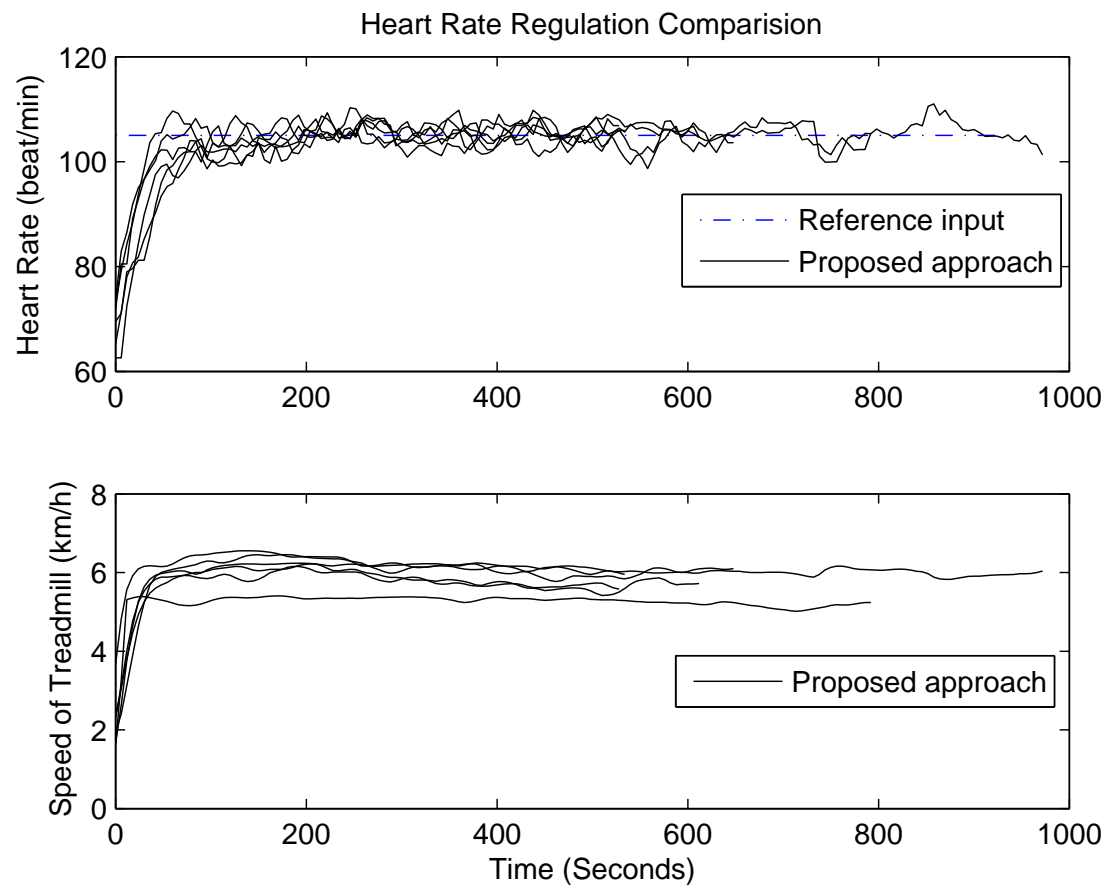

Fig. 11. Step responses for all subjects 

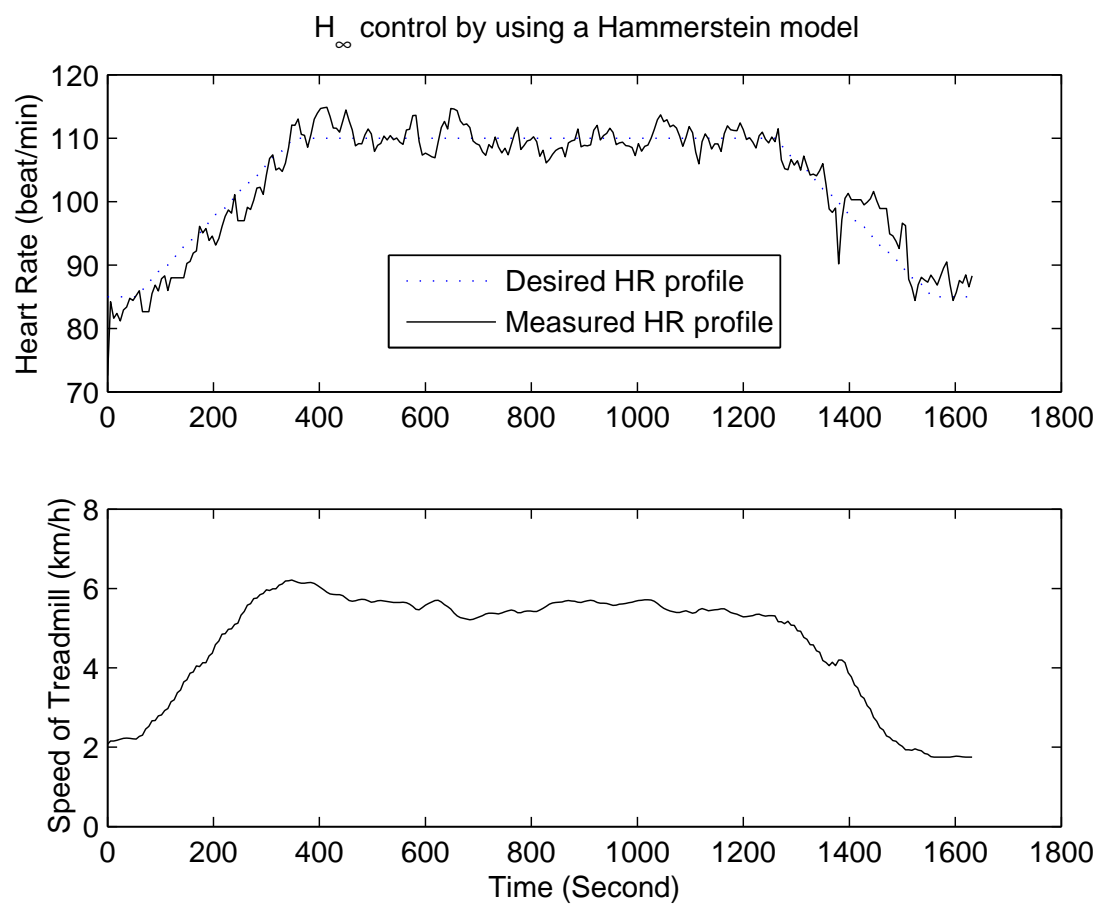

Fig. 12. A typical heart rate tracking for an exercising heart rate profile 

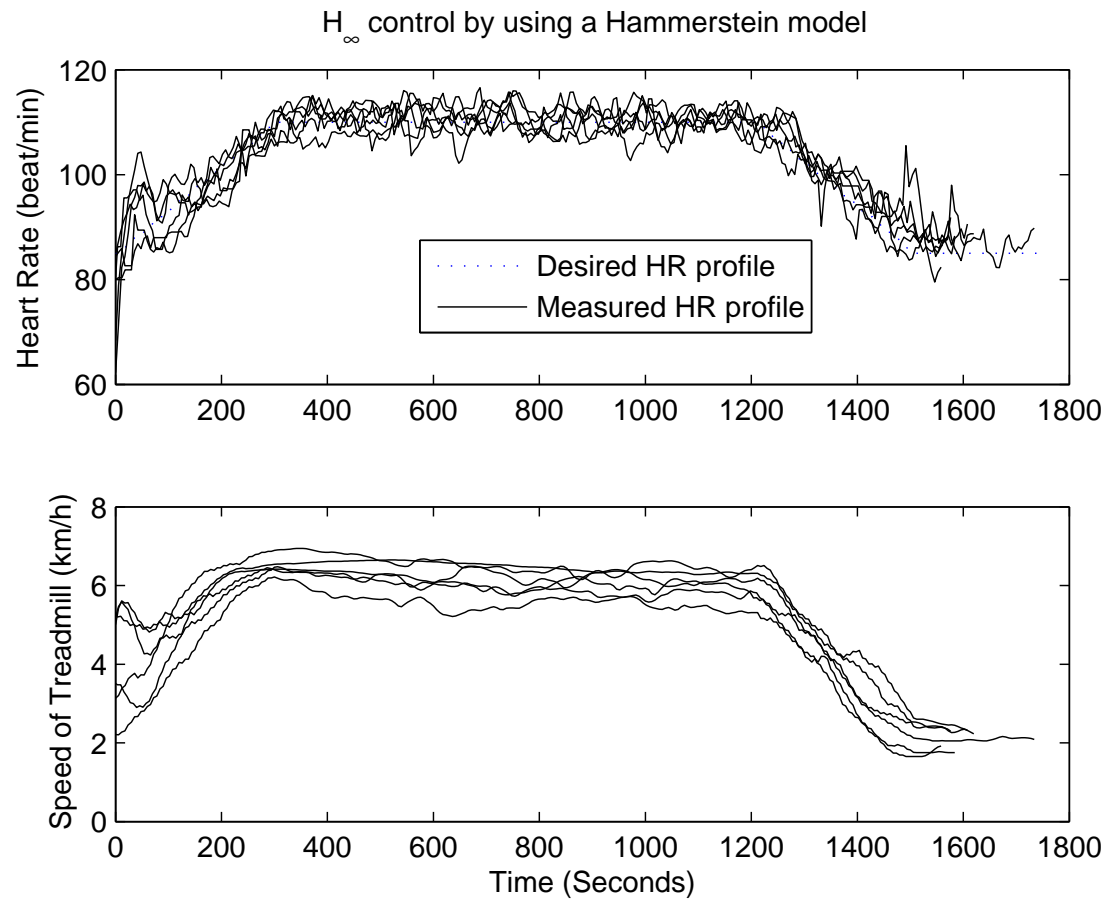

Fig. 13. Heart rate tracking results for all subjects 


\begin{tabular}{cccc}
\hline & Mean & SD & Range \\
\hline Age $(\mathrm{yr})$ & 31.61 & 5.78 & $23-37$ \\
\hline Height $(\mathrm{cm})$ & 176.41 & 5.48 & $169-184$ \\
\hline Body mass $(\mathrm{kg})$ & 74.31 & 9.35 & $60-85$ \\
\hline
\end{tabular}

TABLE I

SUBJECT CHARACTERISTICS $(\mathrm{N}=6)$ 


\begin{tabular}{ccc}
\hline Kernel & Parameter & Regularization Constant C \\
\hline RBF & $\sigma=20.2$ & 5 \\
\hline \hline$\epsilon$-insensitivity & Support vector number & Mean Square Error \\
\hline $0.8 \mathrm{~km} / \mathrm{h}$ & $5(16.7 \%)$ & $0.26(\mathrm{~km} / \mathrm{h})^{2}$ \\
\hline
\end{tabular}

TABLE II

DETAILS ABOUT THE ESTIMATION OF THE INVERSE OF THE NONLINEAR FUNCTION BY SVR 
LIST OF FIGURES

1 A Hammerstein system. . . . . . . . . . . . . . . . . . 2

2 A Hammerstein system. . . . . . . . . . . . . . . . 23

3 The precompensated Hammerstein system . . . . . . . . . . . . . . . . . . . 24

4 A mixed sensitivity $H_{\infty}$ control problem . . . . . . . . . . . . . . 25

5 The computer controlled treadmill system . . . . . . . . . . . . 26

6 Block diagram for computer controlled treadmill system . . . . . . . . . . . . 27

7 Two periods of 31 bits PRBS inputs (treadmill speed) and its corresponding outputs (averaged heart rate of six subjects) . . . . . . . . . . . . 28

8 Modelling of the inverse of the nonlinear function by using $\epsilon$-insensitivity SVR . . 29

9 The triaxial accelerometer monitored two periods of 31 bits PRBS signal . . . . . 30

10 Typical step response comparison between proposed approach and conventional PID

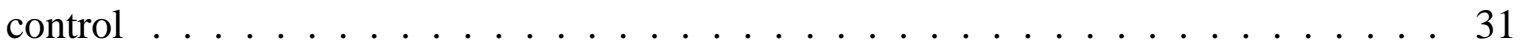

11 Step responses for all subjects . . . . . . . . . . . . . . . 32

12 A typical heart rate tracking for an exercising heart rate profile . . . . . . . 33

13 Heart rate tracking results for all subjects . . . . . . . . . . . . . . 34

\section{LIST OF TABLES}

I Subject characteristics $(\mathrm{N}=6) \ldots \ldots \ldots \ldots \ldots$

II Details about the estimation of the inverse of the nonlinear function by SVR . . . . 36 